\title{
Prediabetes and the risk of cancer: a meta-analysis
}

\author{
Yi Huang • Xiaoyan Cai • Miaozhen Qiu • Peisong Chen • \\ Hongfeng Tang • Yunzhao Hu • Yuli Huang
}

Received: 16 May 2014 / Accepted: 31 July 2014 / Published online: 11 September 2014

(C) Springer-Verlag Berlin Heidelberg 2014

\begin{abstract}
Aims/hypothesis The results from prospective cohort studies of prediabetes (impaired fasting glucose and/or impaired glucose tolerance) and risk of cancer are controversial. We conducted a meta-analysis to evaluate the risk of cancer in association with impaired fasting glucose and impaired glucose tolerance.

Methods The PubMed, EMBASE and Cochrane Library databases were searched for prospective cohort studies with data on prediabetes and cancer. Two independent reviewers assessed the reports and extracted the data. Prospective studies were included if they reported adjusted RRs with $95 \%$ CIs for the association between cancer and prediabetes. Subgroup analyses were conducted according to endpoint, age, sex, ethnicity, duration of follow-up and study characteristics.

Results Data from 891,426 participants were derived from 16 prospective cohort studies. Prediabetes was associated with an increased risk of cancer overall (RR 1.15; 95\% CI 1.06, 1.23).
\end{abstract}

Yi Huang and Xiaoyan Cai contributed equally to this study.

Electronic supplementary material The online version of this article (doi:10.1007/s00125-014-3361-2) contains peer-reviewed but unedited supplementary material, which is available to authorised users.

Y. Huang $\cdot$ X. Cai $\cdot$ H. Tang $\cdot$ Y. Hu $(\bowtie) \cdot$ Y. Huang $(\bowtie)$

Clinical Medicine Research Center, The First People's

Hospital of Shunde, Penglai Road, Daliang Town, Shunde District,

Foshan 528300, People's Republic of China

e-mail: huyunzhao4406@163.com

e-mail: hyuli821@163.com

\section{Qiu}

Department of Medical Oncology, Sun Yat-sen University Cancer Center, State Key Laboratory of Oncology in South China,

Guangzhou, People's Republic of China

P. Chen

Department of Laboratory Medicine, the First Affiliated Hospital of Sun Yat-sen University, Guangzhou, People's Republic of China
The results were consistent across cancer endpoint, age, duration of follow-up and ethnicity. There was no significant difference for the risk of cancer with different definitions of prediabetes. In a site-specific cancer analysis, prediabetes was significantly associated with increased risks of cancer of the stomach/colorectum, liver, pancreas, breast and endometrium (all $p<0.05$ ), but not associated with cancer of the bronchus/ lung, prostate, ovary, kidney or bladder. The risks of sitespecific cancer were significantly different $(p=0.01)$ and were highest for liver, endometrial and stomach/colorectal cancer. Conclusions/interpretation Overall, prediabetes was associated with an increased risk of cancer, especially liver, endometrial and stomach/colorectal cancer.

Keywords Cancer · Impaired fasting glucose $\cdot$ Impaired glucose tolerance $\cdot$ Prediabetes $\cdot$ Risk factors

\section{Abbreviations \\ FPG Fasting plasma glucose \\ IFG Impaired fasting glucose \\ IGT Impaired glucose tolerance \\ NOS Newcastle-Ottawa Quality Assessment Scale}

\section{Introduction}

Prediabetes is a general term that refers to an intermediate stage between normoglycaemia and overt diabetes mellitus. It includes individuals with impaired glucose tolerance (IGT), impaired fasting glucose (IFG) or a combination of the two [1]. In 2003, the ADA redefined the range of fasting plasma glucose (FPG) concentration for diagnosing IFG from 6.1$6.9 \mathrm{mmol} / 1$ to $5.6-6.9 \mathrm{mmol} / 1$ in order to better identify individuals at risk of developing diabetes [2]. However, this change has been contentious and has not been adopted by the 
WHO Expert Group [3] or other international guidelines [4, 5]. One of the main arguments against this change is that it greatly increases the number of individuals labelled as having IFG, without any clear association with clinical complications.

Epidemiological studies have shown that diabetes increases the incidence and mortality of cancer in general, as well as of some specific types of cancer [6, 7]. It has been proposed that cancer should be considered as a new potential complication of diabetes [6]. Some studies have suggested that not only diabetes, but also prediabetes may be associated with an elevated risk of cancer [8-13], although this has not been documented in other studies [14-18]. These inconsistent findings may arise from the use of different definitions and thresholds for prediabetes, non-standard OGTTs, the use of different endpoints (e.g. cancer mortality vs incidence) and small sample sizes.

Considering these inconsistent results, a meta-analysis of prospective cohort studies may help to clarify this issue. Therefore, the objective of the present study was to evaluate the putative association between different definitions of prediabetes and risk of cancer.

\section{Methods}

Search strategy and selection criteria The search strategy was performed in accordance with the recommendations of the Meta-analysis of Observational Studies in Epidemiology Group [19]. Electronic databases (PubMed, EMBASE and the Cochrane Library) were searched for prospective cohort studies to 30 April 2014 using the terms 'blood glucose', 'impaired glucose tolerance', 'impaired fasting glucose', 'prediabetes', 'pre-diabetes', 'prediabetic state', 'hyperglycemia' or 'borderline diabetes' and 'neoplasms', 'neoplasm', 'carcinoma', 'carcinomas', 'carcinomata', 'cancer', 'tumors' or 'tumor'. We restricted the search to human studies. No language restrictions were applied. We also manually reviewed the references cited in potentially relevant studies. The detailed PubMed search strategy is presented in the electronic supplementary material (ESM) Methods. The search strategies were similar for other databases but were adapted where necessary.

Studies were included if they met the following criteria: (1) prospective cohort studies involving participants aged $\geq 18$ years; (2) elevated blood glucose at baseline; (3) a duration of follow-up $\geq 3$ years with an assessment of the risk of cancer; and (4) adjusted RRs and 95\% CIs reported for events associated with prediabetes relative to normoglycaemia. Prediabetes was defined as IFG (FPG in the range either $5.6-6.9 \mathrm{mmol} / 1$ or $6.1-6.9 \mathrm{mmol} / \mathrm{l}$, depending on the study) $[2,20]$ and/or IGT $(2 \mathrm{~h}$ plasma glucose $7.8-11.1 \mathrm{mmol} / 1$ during an OGTT) [3]. Normoglycaemia was defined as an
FPG either $<5.6$ or $<6.1 \mathrm{mmol} / 1$, respectively $[2,20]$, and/or normal glucose tolerance.

Studies were excluded if: (1) enrolment depended on having a particular condition or risk factor (e.g. cardiovascular disease or chronic kidney disease); (2) the reported RR was unadjusted; or (3) the data were derived from the same cohort.

If duplicate studies were derived from the same cohort and reported the same outcome, only the most recent report was included.

Data extraction and quality assessment Two investigators (Y. Huang and X. Cai) conducted independent literature searches using the strategy described above to identify potentially relevant articles. The full manuscripts of potentially relevant studies were obtained and reviewed according to the predefined criteria. Data, including participants' characteristics, duration of follow-up and outcome assessment, were recorded in a standard format. Discrepancies were resolved by discussion with other investigators (M. Qiu and H. Tang). The principal authors of the retrieved studies were contacted for any additional data if required.

We assessed the quality of each study according to the Newcastle-Ottawa Quality Assessment Scale (NOS) for cohort studies [21], in which a study was judged on three broad perspectives as follows: selection (four items, one star each), comparability (one item, up to two stars) and exposure/ outcome (three items, one star each). A star represents a high-quality choice of individual study. In this analysis, a high-quality study was defined as a study with $\geq 7$ stars awarded.

Data synthesis and analysis The primary outcome measure was the risk of all cancer associated with prediabetes. The secondary outcome measure was the RR of site-specific cancer associated with prediabetes. Subgroup analyses of the primary outcome were conducted according to endpoints (cancer mortality vs incidence), ethnicity (Asian vs nonAsian), sex (male vs female), participant's age (mean age $<55$ vs $\geq 55$ years), duration of follow-up ( $<10$ vs $\geq 10$ years), possibility of enrolling patients with diabetes (none enrolled vs might be enrolled) and adjustment of risk factors (age- and sex-adjusted vs multivariate-adjusted).

From each study, we extracted the RR and 95\% CI that were adjusted for potential confounders. We logarithmically transformed these values (to base $e$ ) in each study and calculated the corresponding SEs to stabilise the variance and normalise the distribution [22]. The inverse variance method was used to combine the $\log _{e}$ RRs and SEs using randomeffects models. We used $I^{2}$ statistics to test heterogeneity. Values of $I^{2}>50 \%$ were considered to indicate significant heterogeneity [23]. The test for subgroup differences was calculated by $\chi^{2}$ statistics. We assessed publication bias by inspecting funnel plots for each outcome in which the $\log _{e}$ 
(RR) was plotted against SE, as well as Egger's test (linear regression method).

We also conducted sensitivity analyses in which the pooled RR was recalculated by omitting one study at a time. All $p$ values are two-tailed, and statistical significance was set at 0.05. All analyses were performed with RevMan software version 5.2 for Windows (The Cochrane Collaboration, Copenhagen, Denmark) and Stata version 12.0 (Stata Corp, College Station, TX, USA).

\section{Results}

Studies retrieved and characteristics A total of 13,017 manuscripts were retrieved in the initial search. After screening the abstracts, 67 qualified for a full review. Finally, 16 prospective cohort studies [8-18, 24-28] comprising 891,426 individuals satisfied the study inclusion criteria and were analysed (Fig. 1).

All studies were derived from the general population. Of the 16 studies, four were from Asia [8, 12, 14, 15], 11 were from the USA and Europe [9-11, 13, 17, 18, 24-28], and one was from Africa [16]. Asians accounted for $57.3 \%$ of all participants in the 16 studies. Three studies only enrolled men [12, 24, 25], two studies only enrolled women [9, 26], and the other studies included both men and women. The duration of follow-up ranged from 3.6 to 37 years. Table 1 summarises the key characteristics of the studies included. According to the NOS and predefined quality assessment criteria, all studies were graded as good quality. The details

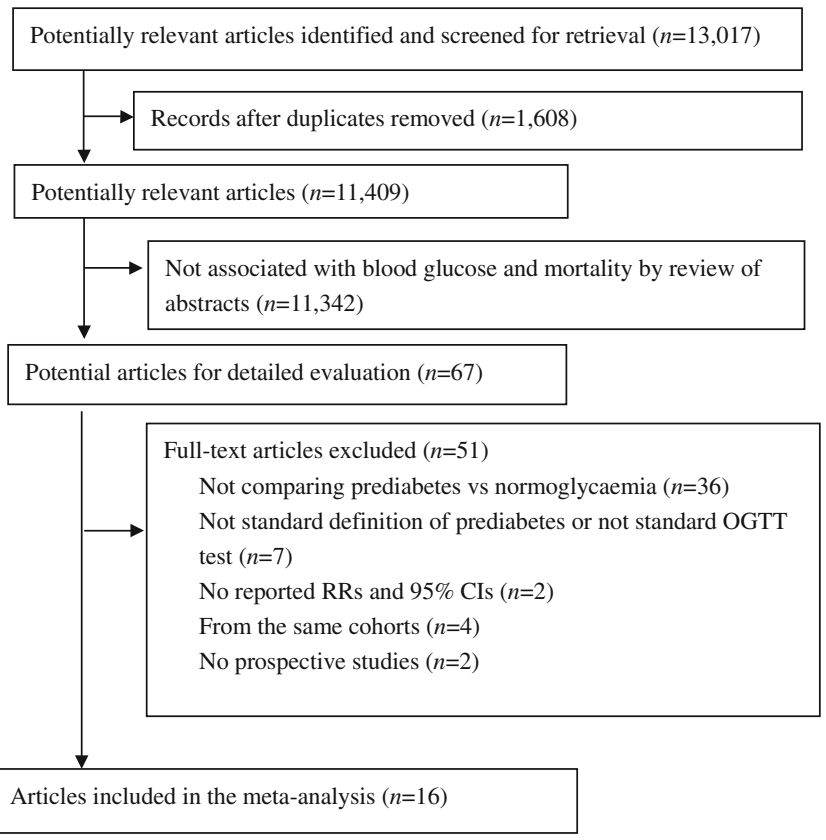

Fig. 1 Flow of papers through the review of the quality assessment are presented in ESM Table 1. All the included studies excluded individuals with an FPG $\geq 7.1 \mathrm{mmol} / 1$. Seven studies only measured FPG at baseline without performing OGTTs; therefore, these studies possibly enrolled patients with a $2 \mathrm{~h}$ plasma glucose $\geq 11.1 \mathrm{mmol} / 1$ [ 9 , $10,12,14,15,25,26]$.

Association between prediabetes and risk of all cancer Five studies reported data for an IFG of 5.6-6.9 mmol/1 [8, 12, 14, $15,26]$, six studies reported data for an IFG of $6.1-6.9 \mathrm{mmol} / 1$ $[9-11,16,18,25]$, six studies reported data for IGT $[8,11,16$, $24,27,28]$, and two studies combined IFG 6.1-6.9 mmol/1 and/or IGT $[13,17]$. No studies assessed the risk of cancer in prediabetes defined as a combined IFG of $5.6-6.9 \mathrm{mmol} / \mathrm{land} /$ or IGT. There was no significant heterogeneity among all the studies or among studies with different definitions of prediabetes (all $I^{2}<50 \%$ ). Therefore, fixed-effects models were used for the analyses.

Prediabetes was associated with an increased risk of cancer overall (RR 1.15; 95\% CI 1.06, 1.23). The risks were also significant when prediabetes was defined as an IFG 5.6$6.9 \mathrm{mmol} / 1$ (RR 1.49 ; 95\% CI 1.05, 2.11), IFG $6.1-$ $6.9 \mathrm{mmol} / \mathrm{l}$ (RR 1.11; 95\% CI 1.01, 1.22), IGT (RR 1.25; $95 \%$ CI $1.02,1.53$ ) or combined IFG $6.1-6.9 \mathrm{mmol} / \mathrm{l}$ and/or IGT (RR 1.11; 95\% CI 1.02, 1.22) (Fig. 2). There was no significant difference for the risk of cancer between different definitions of prediabetes $\left(I^{2}=15.1 \%, p=0.32\right)$. No evidence of publication bias was identified by visual inspection of the funnel plot (Fig. 3) or indicated by Egger's test ( $p=0.95$ ).

Association between prediabetes and the risk of site-specific cancer In the secondary outcome analyses, prediabetes was significantly associated with increased risks of cancer of the stomach/colorectum, liver, pancreas, breast and endometrium (all $p<0.05$ ), but was not associated with cancer of the bronchus/lung, prostate, ovary, kidney or bladder. The risks of sitespecific cancer were significantly different $(p=0.01)$ and were highest for liver, endometrial and stomach/colorectal cancer (Fig. 4).

Subgroup analyses In the subgroup analyses, prediabetes was associated with a significantly higher cancer risk across subgroups conducted according to specific endpoints (cancer mortality vs cancer incidence), ethnicity (Asian vs non-Asian) and duration of follow-up ( $<10$ vs $\geq 10$ years). The relative risk was higher for Asian than non-Asian individuals $(p=0.03)$. Although the associations between prediabetes and risk of cancer were not significant in the subgroups that included participants aged $\geq 55$ years and female participants, were only age- and sex-adjusted, and might have enrolled patients with diabetes, there were no significant differences between comparisons of subgroups (Table 2). 


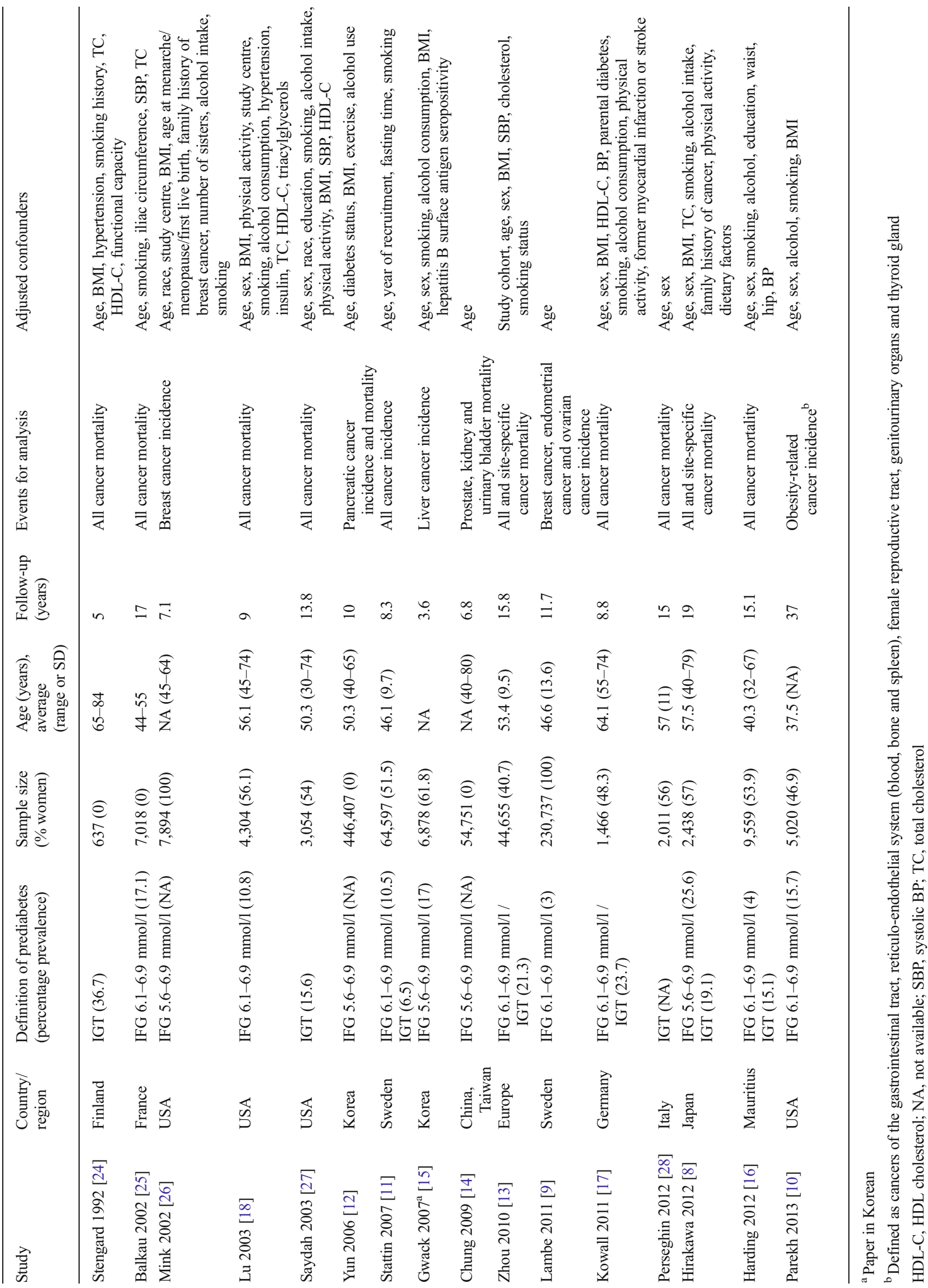


Fig. 2 Forest plot of the comparison between prediabetes and normoglycaemia for an outcome of risk of all cancers. Plotted on a non-linear scale. $\mathrm{IV}$, inverse variance

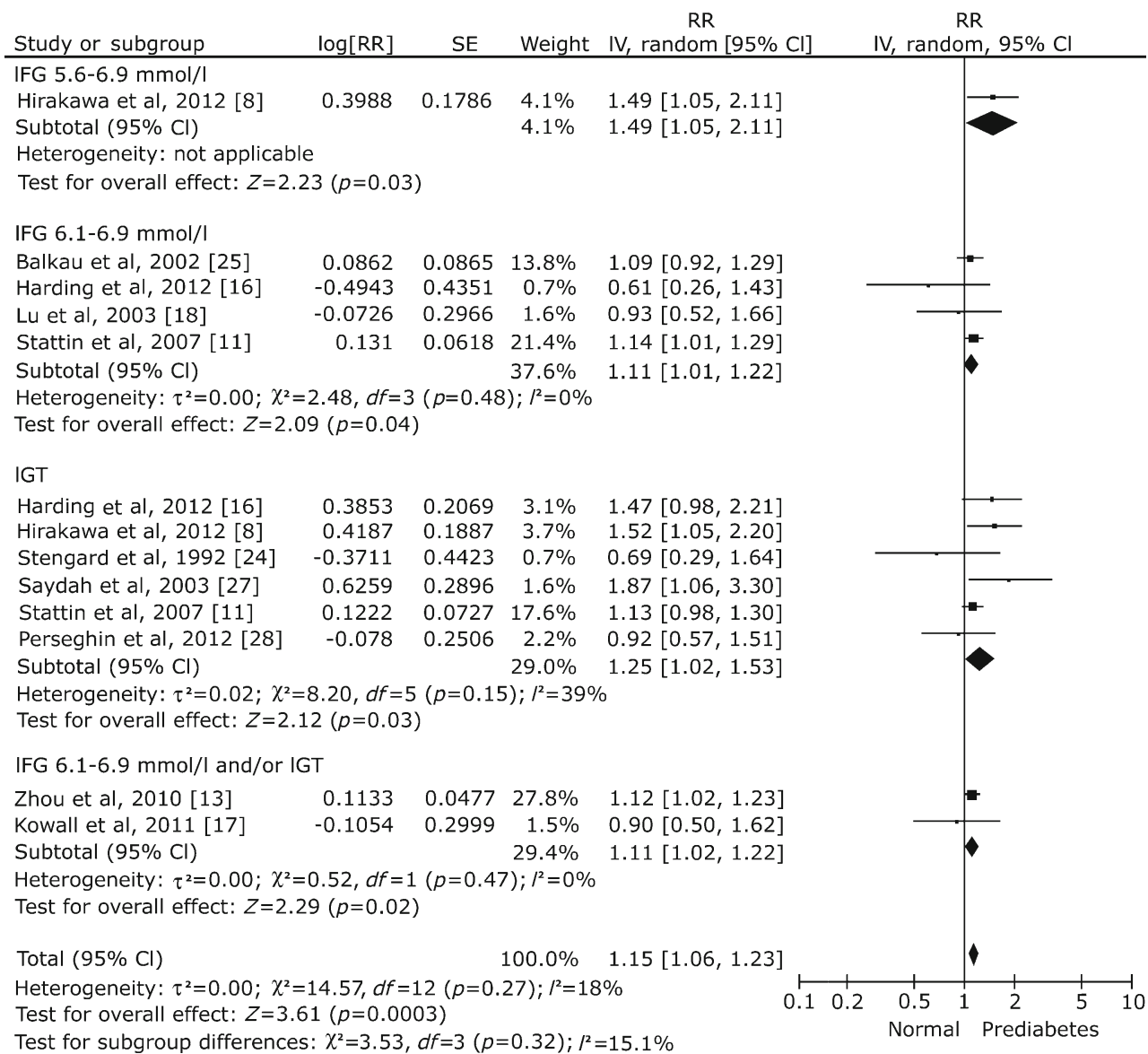

Sensitivity analyses Sensitivity analyses were conducted using several methods, and these analyses confirmed that the primary results were not influenced by the use of fixed-effects models compared with random-effects models, and ORs compared with RRs, or by recalculating the RRs by omitting one study at a time. Furthermore, as it has been reported that obesity, an important risk factor for diabetes, is also linked to the development of cancer, we performed a sensitivity analysis that only included studies that adjusted for BMI in the meta-analysis. We found that, after controlling for BMI,

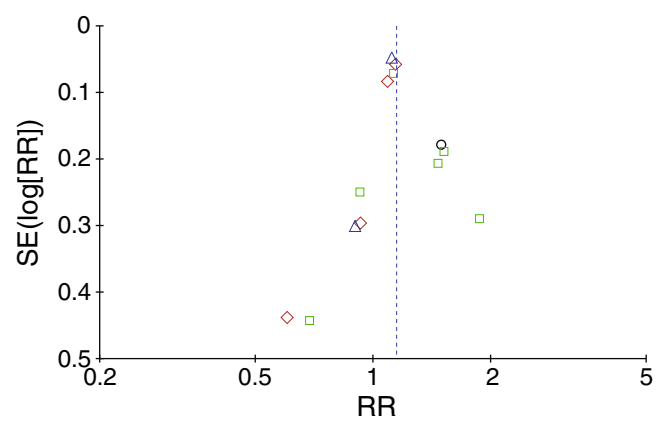

Fig. 3 Funnel plot of the comparison between prediabetes and normoglycaemia for an outcome of risk of all cancers. Subgroups: black circle, IFG 5.6-6.9 mmol/l; red diamonds, IFG 6.1-6.9 mmol/1; green squares, IGT; blue triangles, IFG 6.1-6.9 mmol/1 and/or IGT the presence of prediabetes remained associated with an increased risk of cancer (RR 1.22; 95\% CI 1.01, 1.48) (ESM Fig. 1).

\section{Discussion}

In this meta-analysis of 16 prospective cohort studies comprising more than 890,000 individuals, we found that the presence of prediabetes at baseline was significantly associated with increased risks of cancer in the general population, particularly for liver cancer and stomach or colorectal cancer. The risks were increased when a lower FPG value of 5.6$6.9 \mathrm{mmol} / \mathrm{l}$ was used, according to the current ADA definition of IFG, as well as in participants with IGT. The results were consistent across cancer endpoints, age, study characteristics, follow-up duration and ethnicity.

The lower threshold ADA definition of IFG released in 2003 resulted in a twofold to fivefold increase in the prevalence of IFG in most populations [5]. The lower cut-off point has been controversial [29] and has not been adopted by other guidelines, which have retained the threshold of $6.1-$ $6.9 \mathrm{mmol} / \mathrm{l}[3]$. One of the most important arguments against using the lower threshold is that it greatly increases the 
Fig. 4 Forest plot of the comparison between prediabetes and normoglycaemia for an outcome of site-specific cancer risk. Plotted on a non-linear scale. $\mathrm{IV}$, inverse variance

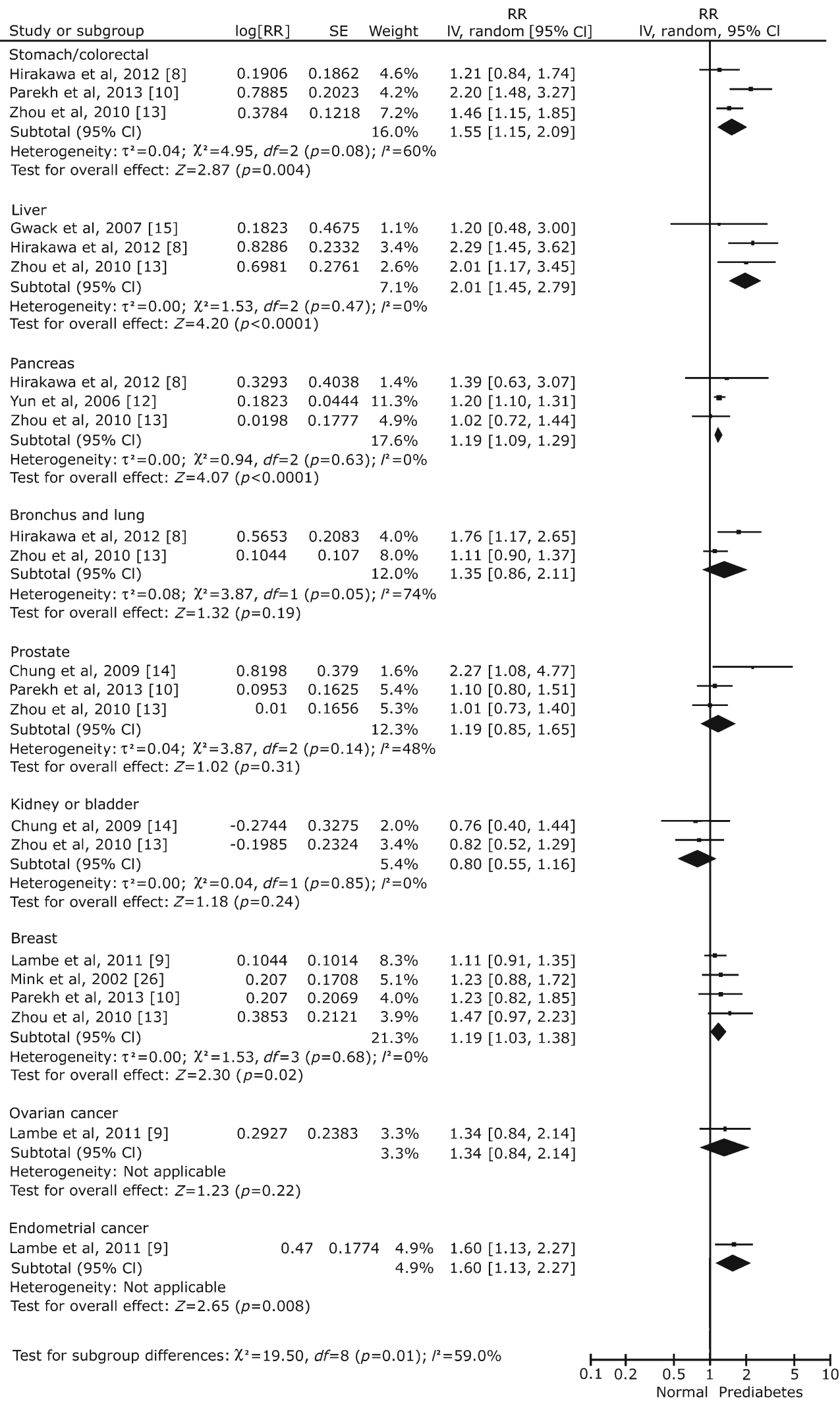

prevalence of IFG, but without enough data to support the clinical prognostic implications [30]. In this study, there was sufficient power to show that prediabetes defined as IFG 5.6-
$6.9 \mathrm{mmol} / \mathrm{l}$, IFG 6.1-6.9 mmol/1, IGT or combined IFG 6.1$6.9 \mathrm{mmol} / \mathrm{l}$ and/or IGT was associated with increased risks of cancer. The results were consistent across specific endpoints, 
Table 2 Subgroup analyses of the association between prediabetes and risk of all cancers

\begin{tabular}{|c|c|c|c|c|}
\hline Subgroup & $\begin{array}{l}\text { Number } \\
\text { of studies }\end{array}$ & RRs (95\% CIs) & $p$ values & $\begin{array}{l}p \text { values } \\
\text { between } \\
\text { subgroups }\end{array}$ \\
\hline \multicolumn{5}{|l|}{ Endpoints } \\
\hline Cancer mortality & 9 & $1.14(1.04,1.25)$ & 0.007 & \multirow[t]{2}{*}{0.73} \\
\hline Cancer incidence & 1 & $1.17(1.03,1.32)$ & 0.01 & \\
\hline \multicolumn{5}{|c|}{ Sex } \\
\hline Men & 5 & $1.17(1.08,1.26)$ & $<0.001$ & \multirow[t]{3}{*}{0.67} \\
\hline Women & 3 & $1.10(0.99,1.22)$ & 0.08 & \\
\hline Both & 8 & $1.15(1.08,1.22)$ & $<0.001$ & \\
\hline \multicolumn{5}{|l|}{ Race/ethnicity } \\
\hline Asian & 1 & $1.50(1.17,1.94)$ & 0.002 & \multirow[t]{2}{*}{0.03} \\
\hline Non-Asian & 9 & $1.12(1.06,1.19)$ & $<0.001$ & \\
\hline \multicolumn{5}{|c|}{ Participant's average age (years) } \\
\hline$<55$ & 5 & $1.14(1.06,1.22)$ & $<0.001$ & \multirow[t]{2}{*}{0.92} \\
\hline$\geq 55$ & 5 & $1.15(0.90,1.47)$ & 0.20 & \\
\hline \multicolumn{5}{|c|}{ Follow-up duration (years) } \\
\hline$<10$ & 4 & $1.12(1.02,1.22)$ & 0.01 & \multirow[t]{2}{*}{0.34} \\
\hline$\geq 10$ & 6 & $1.21(1.06,1.39)$ & $<0.001$ & \\
\hline \multicolumn{5}{|c|}{ Adjustment for risk factors } \\
\hline Age and sex & 1 & $0.92(0.57,1.51)$ & 0.76 & \multirow[t]{2}{*}{0.36} \\
\hline $\begin{array}{l}\text { Multivariate- } \\
\text { adjusted }\end{array}$ & 9 & $1.17(1.08,1.25)$ & $<0.001$ & \\
\hline \multicolumn{5}{|c|}{ Possibility of enrolling patients with diabetes } \\
\hline Yes & 1 & $1.09(0.92,1.29)$ & 0.32 & \multirow[t]{2}{*}{0.53} \\
\hline No & 9 & $1.16(1.06,1.26)$ & $<0.001$ & \\
\hline
\end{tabular}

ethnicities and durations of follow-up, and after adjustment for potential confounders. More importantly, the risks of cancer were very similar with different definitions of prediabetes. These findings support the lower threshold definition IFG proposed by the ADA, and highlight the clinical value of the early management of hyperglycaemia to prevent cancer.

Several mechanisms may be involved in the association between prediabetes and cancer risk. First, chronic hyperglycaemia and its related conditions, such as chronic oxidative stress and the accumulation of advanced glycation end-products, may act as carcinogenic factors [31]. It has been reported that diabetes is associated with an increased production of reactive oxygen species and greater oxidative damage to DNA $[32,33]$. Recently, it has also been reported that the overall frequency of DNA damage and cytotoxicity correlates with the level of $\mathrm{HbA}_{1 \mathrm{c}}$ in people with prediabetes [34].

Second, insulin resistance is a core defect responsible for the development of diabetes, and is established in individuals with prediabetes [35]. The compensatory hyperinsulinaemia and increased level of bioavailable IGF 1 related to insulin resistance may promote the proliferation of cancer cells and may also relate to worsened cancer outcomes [13].

Third, genetic 'interferences' may also play an important role in the development of cancer in prediabetic individuals. A recent study has suggested that nuclear receptor coactivator 5 is a haploinsufficient tumour suppressor, and that a deficiency of nuclear receptor coactivator 5 increases susceptibility to both glucose intolerance and hepatocellular carcinoma, partially by increasing IL-6 expression [36]. In contrast, epidemiological studies have suggested that men with diabetes are less likely to develop prostate cancer than non-diabetic men [37], possibly because of a genetic mechanism linked to the $H N F 1 \beta$ gene (also known as TCF2), which predisposes to diabetes but may protect men from prostate cancer [38]. It is interesting that in our study, prediabetes was most strongly associated with liver cancer, but not associated with prostate, kidney or bladder cancer. Although these results are unlikely to completely explain the epidemiological association between prediabetes and site-specific cancer, they provide a new insight into a possible direct causal link.

These findings have important clinical and public health implications. In the US population aged $\geq 18$ years, the ageadjusted prevalence of prediabetes increased from $29.2 \%$ in 1999-2002 to $36.2 \%$ in 2007-2010 [39]. Considering the high prevalence of prediabetes, as well as the robust and significant association between prediabetes and cancer demonstrated in our study, successful intervention in this large population could have a major public health impact. The ADA suggest that lifestyle intervention is the mainstay of treatment for prediabetes in the general population, and metformin is recommended for delaying progression to overt diabetes if individuals present with other related risk factors, such as a BMI $\geq 35 \mathrm{~kg} / \mathrm{m}^{2}$, dyslipidaemia, hypertension, a family history of diabetes or an $\mathrm{HbA}_{1 \mathrm{c}}>6 \%(42 \mathrm{mmol} / \mathrm{mol})$ [40]. It should be noted that metformin is now considered as having some 'protective' anticancer properties. Notably, metformin mediates an approximately $30 \%$ reduction in the lifetime risk of cancer in diabetic patients [41]. However, whether this is true in prediabetic individuals is not yet known. Long-term, largescale studies of high-risk individuals, especially those with IGT or a combination of IGT and IFG, are urgently needed to explore the effects of metformin interventions on the risk of cancer in people with prediabetes.

The main strengths of this meta-analysis are its very large sample size, with more than 890,000 participants. Furthermore, we only included prospective cohort studies that reported adjusted RRs. Prediabetes is associated with obesity and overweight [1], which are also acknowledged as risk factors for cancer. In the sensitivity analyses, we found that, in studies that adjusted for BMI, prediabetes remained associated with an increased risk of cancer.

This meta-analysis has some limitations. First, individuals with prediabetes are more likely to progress to diabetes than those with normoglycaemia, but most of the studies included did not adjust for subsequent blood glucose levels or interventions. Nevertheless, our study indicates that, on the basis of a snapshot blood glucose measurement, prediabetes is associated with an increased risk of cancer. Second, the 
ADA recently recommended an $\mathrm{HbA}_{1 \mathrm{c}}$ of $5.7-6.4 \%$ (39$46 \mathrm{mmol} / \mathrm{mol}$ ) as another diagnostic marker for prediabetes [42]. We did not include this criterion in our study because few of the retrieved articles reported it. Future prospective cohort studies that include testing of $\mathrm{HbA}_{1 \mathrm{c}}$ may provide more information on the association between prediabetes and cancer. Third, the adjusted confounders in the studies included were inconsistent. The heterogeneity in adjustment is a potential source of bias in our study. The number of studies included in subgroup analyses and the site-specific cancer analyses was limited, and there was significant heterogeneity in some of the subgroups. Finally, although we found that, after controlling for BMI, the presence of prediabetes remained associated with an increased risk of cancer, residual confounding by body fat distribution (instead of overall fatness reflected by BMI) still, however, remains.

In conclusion, this meta-analysis revealed that prediabetes was associated with an increased risk of cancer. The risk increased in people with an FPG as low as $5.6 \mathrm{mmol} / \mathrm{l}$. These results reaffirm the importance of screening for prediabetes using the ADA criteria, with a view to cancer prevention. This information is important to health professionals and those engaged in the prevention of cancer.

Funding The project was supported by the Medical Scientific Research Grant of Health Ministry of Guangdong province, China (No: B2011310, A2012663, B2012343), Scientific Research Fund of Foshan, Guangdong, China (No: 201208227, 201208210) and Scientific Research Fund of Shunde, Guangdong, China (No: 201208210).

Duality of interest The authors declare that there is no duality of interest associated with this manuscript.

Contribution statement $\mathrm{YiH}$ contributed to the design, analysis and interpretation of the data, drafted the article and approved the final version. XC contributed to the conception, design, analysis and interpretation of the data, critical revision of article and final approval. MQ contributed to the design and analysis of the data, critical revision and final approval. PC contributed to the design and analysis of the data, critical revision and final approval. HT and YunH contributed to analysis of the data, critical revision and final approval. YulH contributed to the conception, design, analysis and interpretation of the data and the critical revision, and undertook revisions and final approval of article. YulH is the guarantor of this work.

\section{References}

1. DeFronzo RA, Abdul-Ghani M (2011) Assessment and treatment of cardiovascular risk in prediabetes: impaired glucose tolerance and impaired fasting glucose. Am J Cardiol 108:3B-24B

2. Expert Committee on the Diagnosis and Classification of Diabetes Mellitus (2003) Report of the expert committee on the diagnosis and classification of diabetes mellitus. Diabetes Care 26(Suppl 1):S5-S20

3. World Health Organization (WHO) Consultation (2006) Definition and diagnosis of diabetes and intermediate hyperglycaemia. www.
who.int/diabetes/publications/Definition $\% 20$ and $\% 20$ diagnosis $\%$ 20of\%20diabetes_new.pdf. Accessed 1 Jan 2014

4. Ryden L, Grant PJ, Anker SD et al (2013) ESC Guidelines on diabetes, pre-diabetes, and cardiovascular diseases developed in collaboration with the EASD: the Task Force on diabetes, pre-diabetes, and cardiovascular diseases of the European Society of Cardiology (ESC) and developed in collaboration with the European Association for the Study of Diabetes (EASD). Eur Heart J 34:3035-3087

5. Forouhi NG, Balkau B, Borch-Johnsen K et al (2006) The threshold for diagnosing impaired fasting glucose: a position statement by the European Diabetes Epidemiology Group. Diabetologia 49:822-827

6. Buysschaert M, Sadikot S (2013) Diabetes and cancer: a 2013 synopsis. Diabetes Metab Syndr 7:247-250

7. Seshasai SR, Kaptoge S, Thompson A et al (2011) Diabetes mellitus, fasting glucose, and risk of cause-specific death. N Engl J Med 364: 829-841

8. Hirakawa Y, Ninomiya T, Mukai N et al (2012) Association between glucose tolerance level and cancer death in a general Japanese population: the Hisayama Study. Am J Epidemiol 176:856-864

9. Lambe M, Wigertz A, Garmo H, Walldius G, Jungner I, Hammar N (2011) Impaired glucose metabolism and diabetes and the risk of breast, endometrial, and ovarian cancer. Cancer Causes Control 22: $1163-1171$

10. Parekh N, Lin Y, Vadiveloo M, Hayes RB, Lu-Yao GL (2013) Metabolic dysregulation of the insulin-glucose axis and risk of obesity-related cancers in the Framingham Heart Study-offspring cohort (1971-2008). Cancer Epidemiol Biomarkers Prev 22:18251836

11. Stattin P, Bjor O, Ferrari P et al (2007) Prospective study of hyperglycemia and cancer risk. Diabetes Care 30:561-567

12. Yun JE, Jo I, Park J et al (2006) Cigarette smoking, elevated fasting serum glucose, and risk of pancreatic cancer in Korean men. Int J Cancer 119:208-212

13. Zhou XH, Qiao Q, Zethelius B et al (2010) Diabetes, prediabetes and cancer mortality. Diabetologia 53:1867-1876

14. Chung H, Chen S, Li M (2009) Diabetes and risk of death from cancer of the prostate, kidney, and urinary bladder. Urology 74:S36S37

15. Gwack J, Hwang SS, Ko KP et al (2007) Fasting serum glucose and subsequent liver cancer risk in a Korean prospective cohort. J Prev Med Public Health 40:23-28 [article in Korean]

16. Harding JL, Soderberg S, Shaw JE et al (2012) All-cause cancer mortality over 15 years in multi-ethnic Mauritius: the impact of diabetes and intermediate forms of glucose tolerance. Int J Cancer 131:2385-2393

17. Kowall B, Rathmann W, Heier M et al (2011) Categories of glucose tolerance and continuous glycemic measures and mortality. Eur J Epidemiol 26:637-645

18. Lu W, Resnick HE, Jain AK et al (2003) Effects of isolated postchallenge hyperglycemia on mortality in American Indians: the Strong Heart Study. Ann Epidemiol 13:182-188

19. Stroup DF, Berlin JA, Morton SC et al (2000) Meta-analysis of observational studies in epidemiology: a proposal for reporting. Meta-analysis Of Observational Studies in Epidemiology (MOOSE) group. JAMA 283:2008-2012

20. The Expert Committee on the Diagnosis and Classification of Diabetes Mellitus (1997) Report of the Expert Committee on the Diagnosis and Classification of Diabetes Mellitus. Diabetes Care 20: 1183-1197

21. Wells GA, Shea B, O'Connell D et al (2003) The Newcastle-Ottawa Scale (NOS) for assessing the quality of nonrandomised studies in meta-analyses. http://www.ohri.ca/programs/clinical_epidemiology/ oxford.asp. Accessed 1 Jan 2014

22. Lee M, Saver JL, Chang KH, Liao HW, Chang SC, Ovbiagele B (2010) Low glomerular filtration rate and risk of stroke: meta-analysis. BMJ 341:c4249 
23. Imdad A, Yakoob MY, Bhutta ZA (2011) The effect of folic acid, protein energy and multiple micronutrient supplements in pregnancy on stillbirths. BMC Public Health 11(Suppl 3):S4

24. Stengard JH, Tuomilehto J, Pekkanen J et al (1992) Diabetes mellitus, impaired glucose tolerance and mortality among elderly men: the Finnish cohorts of the Seven Countries Study. Diabetologia 35:760-765

25. Balkau B, Forhan A, Eschwege E (2002) Two hour plasma glucose is not unequivocally predictive for early death in men with impaired fasting glucose: more results from the Paris Prospective Study. Diabetologia 45:1224-1230

26. Mink PJ, Shahar E, Rosamond WD, Alberg AJ, Folsom AR (2002) Serum insulin and glucose levels and breast cancer incidence: the atherosclerosis risk in communities study. Am J Epidemiol 156:349352

27. Saydah SH, Loria CM, Eberhardt MS, Brancati FL (2003) Abnormal glucose tolerance and the risk of cancer death in the United States. Am J Epidemiol 157:1092-1100

28. Perseghin G, Calori G, Lattuada G et al (2012) Insulin resistance/ hyperinsulinemia and cancer mortality: the Cremona study at the 15 th year of follow-up. Acta Diabetol 49:421-428

29. Shaw J (2011) Diagnosis of prediabetes. Med Clin N Am 95:341352

30. De Caterina R, Madonna R (2010) Impaired fasting plasma glucose and long-term cardiovascular risk: still a foggy relationship. Eur Heart J 31:1159-1162

31. Abe R, Yamagishi S (2008) AGE-RAGE system and carcinogenesis. Curr Pharm Des 14:940-945

32. Rehman A, Nourooz-Zadeh J, Moller W, Tritschler H, Pereira P, Halliwell B (1999) Increased oxidative damage to all DNA bases in patients with type II diabetes mellitus. FEBS Lett 448:120-122
33. Tabak O, Gelisgen R, Erman $\mathrm{H}$ et al (2011) Oxidative lipid, protein, and DNA damage as oxidative stress markers in vascular complications of diabetes mellitus. Clin Invest Med 34:E163-E171

34. Pereira CS, Molz P, Palazzo RP et al (2013) DNA damage and cytotoxicity in adult subjects with prediabetes. Mutat Res 753:76-81

35. Faerch K, Borch-Johnsen K, Holst JJ, Vaag A (2009) Pathophysiology and aetiology of impaired fasting glycaemia and impaired glucose tolerance: does it matter for prevention and treatment of type 2 diabetes? Diabetologia 52:1714-1723

36. Gao S, Li A, Liu F et al (2013) NCOA5 haploinsufficiency results in glucose intolerance and subsequent hepatocellular carcinoma. Cancer Cell 24:725-737

37. Zhang F, Yang Y, Skrip L et al (2012) Diabetes mellitus and risk of prostate cancer: an updated meta-analysis based on 12 case-control and 25 cohort studies. Acta Diabetol 49(Suppl 1):S235-S246

38. Frayling TM, Colhoun H, Florez JC (2008) A genetic link between type 2 diabetes and prostate cancer. Diabetologia 51: $1757-1760$

39. Bullard KM, Saydah SH, Imperatore G et al (2013) Secular changes in U.S. prediabetes prevalence defined by hemoglobin A1c and fasting plasma glucose: National Health and Nutrition Examination Surveys, 1999-2010. Diabetes Care 36:2286-2293

40. Nathan DM, Davidson MB, DeFronzo RA et al (2007) Impaired fasting glucose and impaired glucose tolerance: implications for care. Diabetes Care 30:753-759

41. Leone A, Di Gennaro E, Bruzzese F, Avallone A, Budillon A (2014) New perspective for an old antidiabetic drug: metformin as anticancer agent. Cancer Treat Res 159:355-376

42. American Diabetes Association (2013) Diagnosis and classification of diabetes mellitus. Diabetes Care 36(Suppl 1):S67-S74 